\section{A computer program for randomization tests for predicted trends}

\section{EUGENE S. EDGINGTON and ALLAN R. STRAIN University of Calgary, Calgary, Alberta, Canada T2N $1 N 4$}

When experimental treatments vary quantitatively along a dimension, experimenters may want to test hypotheses with tests sensitive to linear, quadratic, or other trends in the measurements over experimental treatments. Standard parametric trend analysis is sometimes used for that purpose, but the probability tables are based on a random-sampling model. This makes them inappropriate for the typical psychological experiment, in which there is random assignment to treatments but not random selection of subjects.

A computer program for randomization tests for trend for both independent groups and repeatedmeasures designs has been written. The user can employ an exact randomization test that is based on all divisions of the data or, when the number of divisions is very large, an approximate randomization test based on a random sample of all possible divisions selected by a random number generator routine. An approximate randomization test is a perfectly valid test and is almost as powerful as an exact test (Edgington, 1969).

The randomization tests for predicted trends test the hypothesis that all levels of the independent variable have the same effect, that is, the null hypothesis of no treatment effect. Just as a predicted direction of difference can be incorporated into a $t$ test to make it more sensitive, so can predicted trends make a test of a difference between treatments more sensitive when there are more than two treatments. Very specific predictions can be utilized. For example, instead of just predicting a linear trend, an experimenter can predict a linear downward trend if he expects the response measures to drop for higher values of the independent variable. Or, instead of simply predicting a quadratic trend, the experimenter. can predict a U-shaped quadratic trend of response measures and the value of the independent variable about which the response measures will be symmetrically distributed. Any standard mathematical functional relationship can be predicted. Also, the experimenter can use predicted trends that are not readily specifiable by any of the common mathematical functions, such as a trend in data from an earlier experiment that is being replicated.

The experimenter indicates his predicted trend by providing numerical values ("coefficients"). Each coefficient is transformed into an expected mean value. The test statistic is

$$
\mathrm{n}_{1}\left(\overline{\mathrm{X}}_{1}-\mathrm{EM}_{1}\right)^{2}+\ldots+\mathrm{n}_{\mathrm{k}}\left(\overline{\mathrm{X}}_{\mathrm{k}}-\mathrm{EM}_{\mathrm{k}}\right)^{2}
$$

where $n_{k}$ is the number of subjects taking the kth treatment, $\bar{X}_{k}$ is the mean of the scores for the kth treatment, and $\mathrm{EM}_{k}$ is the expected mean for the kth treatment. The test statistic is the weighted sum of squared deviations of the means (for any particular division of the data) from the corresponding expected means. The probability value (significance) associated with the experimental results is the proportion of the data divisions (randomizations) that provide a test statistic as small as the value associated with the obtained results.

Randomization tests for trend are discussed in more detail elsewhere (Edgington, 1975), and examples of the application of such tests to experimental data can be found in an article by Price and Cooper (1975).

Limitations. The program is limited to 20 treatments and a maximum of 1000 data points.

Input. The setup for an analysis is as follows(1) Title and parameter card: columns $1-40=$ title; columns $41-45=$ number of treatments; columns $46-50$ = number of data points per card, if repeated measures; for independent groups analysis, leave space blank; columns $51.55=$ any nonzero digit for performing an exact randomization test. (2) Size of groups card: columns $1-4=$ number of scores for Treatment 1 ; columns $5-8=$ number of scores for Treatment 2 , etc. (3) Coefficients card: columns $1-4=$ coefficient for Treatment 1 ; columns $5-8=$ coefficient for Treatment 2 , etc. (4) Input format card: e.g., (F4.1). (5) Data deck.

Output. The computer output for the program includes the title, the mean for each treatment, and the significance or probability value.

Language and Computer. The program is written in FORTRAN IV and has been tested on a CDC Cyber 70 . Because the random number function may not be standard for all systems using FORTRAN, the user should check his system for the random number function to call.

Availiability. A source listing and user's manual, which includes sample problems, can be obtained free by writing E.S. Edgington, Department of Psychology, University of Calgary, Calgary, Alberta, Canada.

\section{REFERENCES}

Edgington, E. S. Approximate randomization tests. Journal of Psychology, 1969, 72, 143-149.

Edgington, E. S. Randomization tests for trends. Canadian Psychological Review, 1975, 16, 49-53.

Price, M. T. C., \& Cooper, R. M. U-shaped functions in a shock-escape task. Journal of Comparative and Physiological Psychology, 1975, 89, 600-606. 